\title{
A new amplitude-frequency formula for non-conservative oscillators
}

\author{
Andres Garcia ${ }^{1}$ \\ ${ }^{1}$ Universidad Tecnológica Nacional
}

November 11, 2020

\begin{abstract}
This paper formalize the existence's proof of first-integrals for any second order ODE, allowing to discriminate periodic orbits. Up to the author's knowledge, such a powerful result is not available in the literature providing a tool to determine periodic orbits/limit cycles in the most general scenario.
\end{abstract}

\section{Hosted file}

A_T_Garcia.pdf available at https://authorea.com/users/374830/articles/492260-a-newamplitude-frequency-formula-for-non-conservative-oscillators 